\title{
Die Runeninschrift auf dem Rinderknochen von Břeclav, Flur Lány (Südmähren, Tschechische Republik)
}

\author{
Jiří Macháček und Robert Nedoma \\ Masarykova univerzita | Universität Wien
}

\begin{abstract}
During recent excavations at Břeclav-Lány (southern Moravia, Czech Republic), archaeologists have found a fragmented bovine rib with runes. The rib was unearthed in an early Slavic pit-house and is radiocarbondated to ca. 6oo. The inscription begins at the break line and reads xbemdo (probably tbemdo), representing six of the last eight runes of the older fupark - it seems that the lost piece of the rib exhibited the preceding part of the rune row. There is reason to believe that the carver was a Langobard who did not join the migration into northern Italy in 568 (or, alternatively, a Slav who learned and used the Germanic script?).
\end{abstract}

\section{Archäologischer Befund (Jiř́i Macháček)}

Bei Grabungen in den Jahren 2015 bis 2017 wurden in der Stadt Břeclav (Südmähren, CZ) in der Flur Lány, die in der Nähe der Mündung der Dyje (Thaya) in die Morava (March) im Dreiländereck von Österreich, Tschechien und der Slowakei gelegen ist, insgesamt 37 zumeist eingetiefte Siedlungsobjekte entdeckt. Davon stammen 26 eingetiefte Siedlungsobjekte samt 5 Grubenhäusern, 2 Vorratsgruben und einem ebenerdigen Befund aus dem Zeitraum vom 6. bis zum 8./9. Jahrhundert. Diese Datierung fußt zum einen auf awarenzeitlichen Bronzegegenständen des 8. Jahrhunderts, zum anderen und vor allem auf dem Keramikfundgut (Scherben von Töpfen bzw. topfartigen Gefäßen) in einer für die Prager Kultur des 6.-7. Jahrhunderts charakteristischen Form. Weitere Merkmale der Prager Kultur, die sich auf Südmähren und Niederösterreich bis zum Donautal erstreckt hat, sind Brandbestattung und Grubenhäuser mit einem Steinofen in der Ecke. In den Repräsentanten der Prager Kultur sieht man frühe Slawen (Jelínková 1985:466; Dostál 1993; Szameit 2000; Wawruschka 2009; Nowotny 2015: 99-102); als der 
Prager Kultur zugehörig bzw. als frühslawisch kann etwa ein Drittel der Siedlungsobjekte von Lány bestimmt werden.

In der 2017 aufgedeckten Siedlungsgrube Nr. 25, die die ältesten Keramikobjekte des Prager Typus aus der Siedlung von Lány enthielt, wurden verschiedene Tierknochen gefunden. Nach der Reinigung und der Laborbehandlung wurden auf der ventralen Seite einer abgebrochenen Rinderrippe (Institut für Archäologie und Museologie, Masaryk-Universität Brno, Inv.-Nr. BL 15.829; Maße: 107 mm Länge, $26 \mathrm{~mm}$ Breite) Runenritzungen entdeckt. Proben des Knochens wurden im Radiokarbonlabor der Adam-Mickiewicz-Universität Poznan (Posen) einer Radiokarbon- und einer DNA-Analyse unterzogen. Dank eines hohen Kollagen-Anteils (7,1\% coll) konnten die Proben (Poz-99473) mit Erfolg datiert werden. Das ${ }^{14} \mathrm{C}$-Alter des Knochens beträgt $1455 \pm 30$ BP; nach der Kalibrierung ergab sich auf Wahrscheinlichkeitsniveau 68,2\% die kalendarische Zeitspanne $585 \mathrm{AD}-640 \mathrm{AD}$, auf Wahrscheinlichkeitsniveau 95,4\% die kalendarische Zeitspanne 555 AD-650 AD. Das Rind fand also in der 2. Hälfte des 6. Jahrhunderts oder in der 1. Hälfte des 7. Jahrhunderts den Tod, wahrscheinlich um die Jahrhundertwende.

Zur Bestätigung der Zeitbestimmung wurden noch weitere zwei Rinderknochen ohne Inschrift aus Siedlungsgrube Nr. 25 radiokarbondatiert. Die erbrachten Daten (Poz-98266: 1505 \pm 30 BP, Poz-98267: $1510 \pm 30$ BP) sichern die Datierung von Grube und Grubenfüllung in die 2. Hälfte des 6. Jahrhunderts (nach der Kalibrierung für Poz-98266 540 AD-601 AD bei 68,2\% Wahrscheinlichkeitsniveau, für Poz-98267 536 AD-604 AD bei 68,2\% Wahrscheinlichkeitsniveau). Bei diesen beiden Rinderknochen handelt es sich um die ältesten zuverlässig datierten frühslawischen Funde auf tschechischem Gebiet. Der Runenknochen lag im oberen Bereich der Füllung von Grube Nr. 25 und gelangte offenbar erst in der letzten Phase der Grubenzuschüttung dorthin.

\section{Runeninschrift (Robert Nedoma)}

Die Ritzungen befinden sich auf der ventralen, glatten Seite der nur teilweise erhaltenen Rinderrippe von Břeclav. Die ungleichmäßig angebrachten Runen laufen von links nach rechts und sind ca. 16 bis $20 \mathrm{~mm}$ hoch; die Runenfolge erstreckt sich über ca. $50 \mathrm{~mm}$. Die Beschreibfläche ist von einer Vielzahl von Kratzern übersät. Nach einer gemeinsamen Autopsie mit Klaus Düwel (2.12.2017) ergibt sich folgende Lesung:

Rune Nr. 1: Von den am Bruch befindlichen Ritzungen sind der obere Teil eines Stabes und ein von dessen Spitze nach rechts unten führender gebogener Zweig zu erkennen. Eine Ergänzung zu $\uparrow \mathrm{t}$ ist wahrscheinlich (s. unten). - Rune 


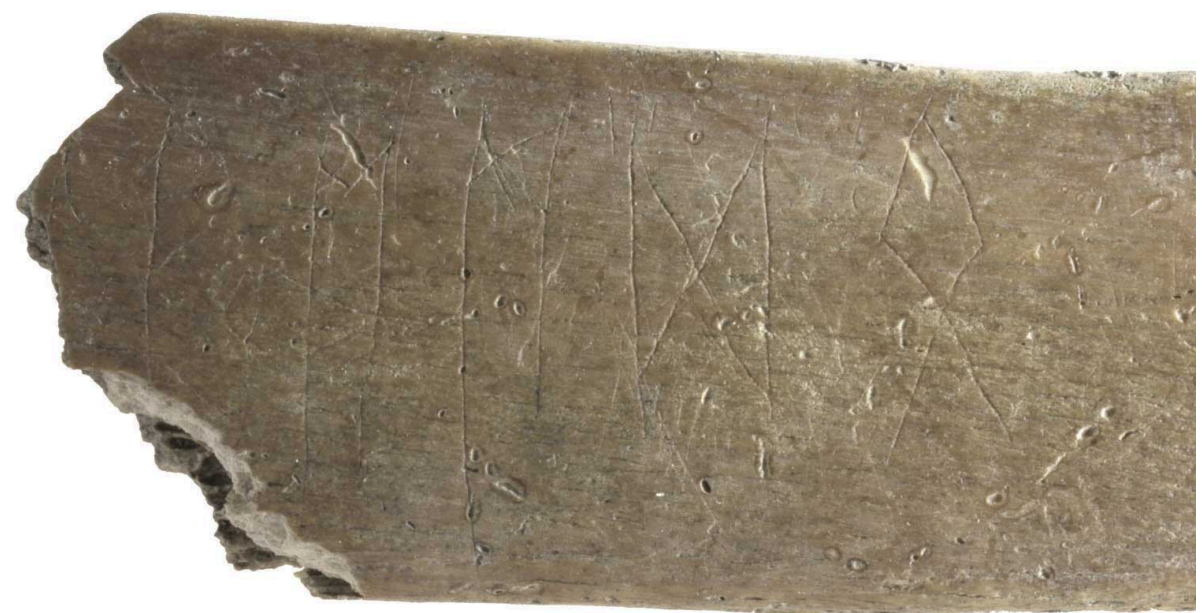

Abbildung 1. Runeninschrift auf dem Rinderknochen von Břeclav (CZ). Foto: Vojtěch Nosek, Institut für Archäologie und Museologie, Masaryk-Universität Brno

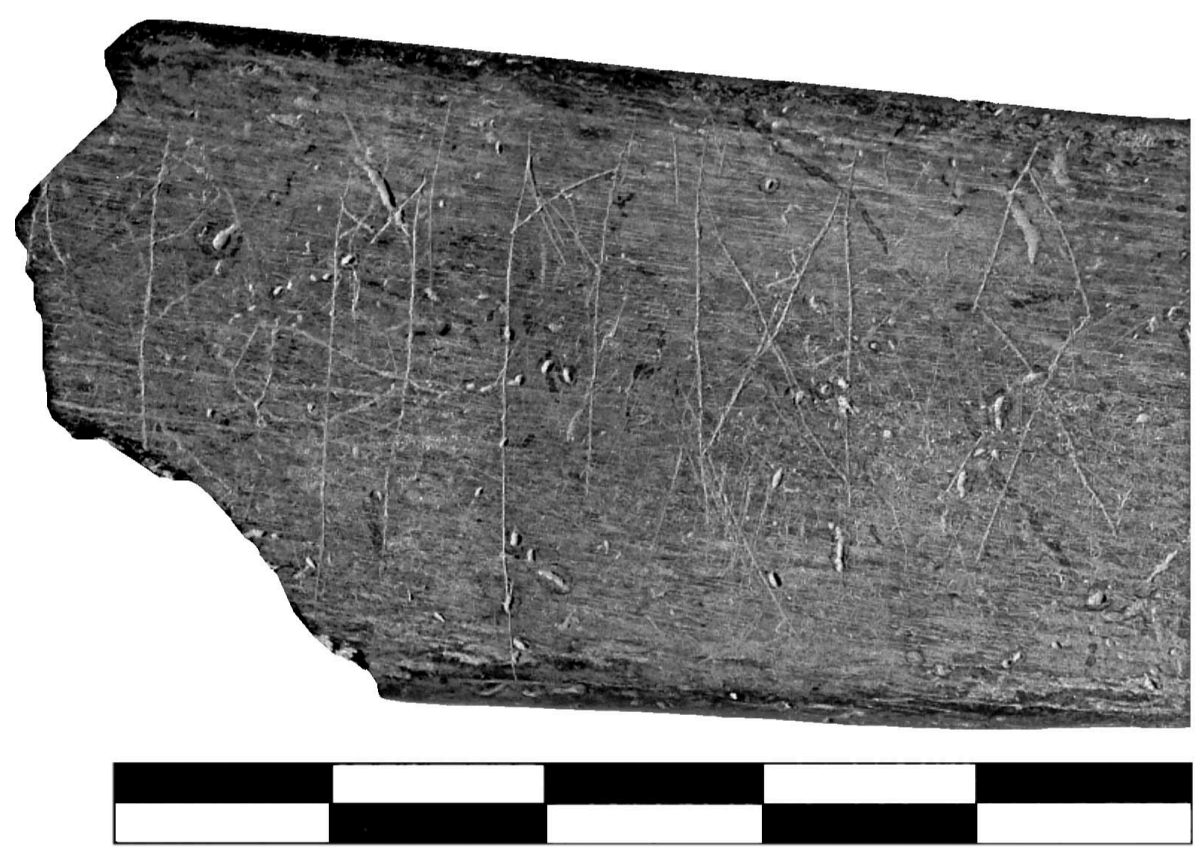

Abbildung 2. Runeninschrift auf dem Rinderknochen von Břeclav (CZ). UV-Aufnahme: Mikoláš Jurda, LAMORFA, Institut für Anthropologie, Masaryk-Universität Brno

Nr. 2 ist ein für die südgermanischen Runeninschriften charakteristisches b mit auseinanderliegenden Haken. Der untere Teil ist abgebrochen, sodaß der Fuß 
des Stabes und der untere Teil des unteren Hakens fehlen. - Rune Nr. 3: M e, dessen linksschräger Zweig unterhalb der Spitze des linken Stabes ansetzt; darüber befindet sich ein tiefer Kratzer. Im unteren Bereich der Runen Nr. 2 und 3 verlaufen zwei parallele Querlinien, die ein den Runenritzungen ganz ähnliches Profil aufweisen; falls es sich um intendierte Gravuren handelt, bleibt deren Funktion unklar. - Rune Nr. 4: $\mathrm{A}$ m, dessen linker Stab länger ist. Beide Diagonalen setzen nicht an den Stabspitzen an, und ihr Schnittpunkt liegt weit im linken Bereich; bei der linksschrägen Diagonale hat der/die Ritzer(in) dreimal angesetzt. - Rune Nr. 5 ist etwas breiter als die vorangehenden Zeichen. Die Diagonalen des $\boldsymbol{W} \mathbf{d}$ erreichen weder die oberen noch die unteren Enden der Stäbe; bei der rechtsschrägen Diagonale ist zweimal angesetzt worden. Zu beiden Seiten des oberen Endes des linken Stabes befinden sich kurze Vertikallinien, deren Funktion unklar ist. Man könnte versucht sein, links ein kleines $\lceil$ (ca. $3 \mathrm{~mm}$ hoch) und rechts ein noch kleineres $Y$ (oder gar $Y$; ca. $2 \mathrm{~mm}$ hoch) zu vermuten; die hypothetischen Zweige sind jedoch flacher und wahrscheinlich nur Materialbeschädigungen. Womöglich deuten die kurzen Vertikallinien darauf, daß der/die Ritzer(in) beim linken Stab dreimal angesetzt hat. - Rune Nr. 6 ist (abgesehen davon, daß die linke Dachlinie ein wenig über den 'First' hinausreicht) ein einigermaßen regelmäßig ausgeführtes $\widehat{\mathbf{x}} \mathbf{o}$.

In den Runen Nr. 2-6 ist die Folge bemdo zu erkennen; bei der unvollständigen Rune Nr. 1 kann es sich um ein $\mathbf{t}$ handeln, sodaß die Sequenz tbemdo sechs der acht Runen aus der letzten $\propto$ ett des älteren Fupark wiedergibt. Warum der/die Ritzer(in) zwischen $\mathbf{m}$ und $\mathbf{d}$ die beiden Runen $\mathbf{l}$ und $\mathbf{y}$ ausgelassen hat, bleibt unklar. Es ist zu vermuten, daß auf der unversehrten Rinderrippe ursprünglich auch die dem $\mathbf{t}$ vorangehenden Runen des älteren Fupark eingeritzt waren und damit eine vollständige Zeichenreihe intendiert war. Der Runenknochen von Břeclav ist der erste bekannte Beleg für das erhaltene Ende des älteren Fupark in den südgermanischen Inschriften; selbst die annähernd vollständige Runenreihe auf der Halbsäule von Breza (SG-19; RäF 95) ${ }^{1}$ bricht nach Rune Nr. 201 ab. Häufiger sind im südgermanischen Corpus Fupark-Abbreviaturen bzw. -Zitate, die stets den Beginn (und nicht das Ende) der Zeichenreihe wiedergeben. ${ }^{2}$

Über die Funktion der Inschrift lässt sich nichts Definitives sagen. Angesichts der wohl fehlerhaften Auslassung von $\mathbf{l}$ und $\mathbf{y}$ wird man am ehesten von einer Schreibübung ausgehen. Magische Zwecke sind zwar nicht ganz auszuschließen,

1. In dieser Inschrift fehlt die $b$-Rune. - Die SG-Nummern beziehen sich auf die demnächst erscheinende Edition von Düwel, Nedoma \& Oehrl (im Druck).

2. Belege: fuparkgw Fibel von Aquincum (SG-2; RäF 7), fupar (plus zj) Fibel von Beuchte (SG-12; RäF 8), fup[a]ṛ[k] Holzstrebe von Trossingen (SG-120); unsicher ist fpa (plus e) Fibel von Herbrechtingen (SG-52; RäF 154). 
aber weniger wahrscheinlich, denn die Operativität der Runenreihe basiert auf Ordnung und (intentioneller) Vollständigkeit. ${ }^{3}$

Ferner befinden sich auf der anderen Seite des Runenknochens von Břeclav zwei kleinere $\times$-artige Gebilde, die flacher sind: allem Anschein nach handelt es sich nicht um $(g$-)Runen.

\section{Fazit (Jiř́i Macháček, Robert Nedoma)}

Nach verbreiteter Ansicht haben die germanischen gentes Mähren und Niederösterreich vor dem Einzug der Slawen verlassen, und zwar spätestens im Zuge der Abwanderung der Langobarden nach Oberitalien im (oder sogar kurz vor dem) Jahr 568. ${ }^{4}$ Auch der Neufund von Břeclav - das bisher einzige authentische Runendenkmal aus Tschechien ${ }^{5}$ und wohl auch das älteste Schriftdokument der ganzen slawischen Welt - lässt Zweifel an einem strikten Nacheinander von Abzug der Langobarden und Zuzug der Slawen entstehen. Angesichts der (wegen des charakteristischen Form der $b$-Rune) sicher südgermanischen Runeninschrift ist zu vermuten, daß eine germanische Restbevölkerung in den mährischen Sitzen der Langobarden zurückgeblieben ist - ob da ein(e) Runenkundige(r) die Strapazen des langen Marsches nach Oberitalien nicht auf sich nehmen wollte oder neugeknüpfte familiäre Bande zu den Slawen ihn/sie in Südmähren zurückhielten? Die Annahme einer langobardischen Inschrift ist wohl die Möglichkeit, die am meisten für sich hat, zumal zumindest drei Runendenkmäler aus dem südgermanischen Corpus den Langobarden zuzusprechen sind. ${ }^{6}$ Alternativ könnte man auch an Schrifttransfer denken und die Runenritzung einem/einer in der Siedlung von Lány ansässigen Slawen/-in zuschreiben; dies wäre allerdings der einzige Fall von Kenntnis und Gebrauch germanischer Runen durch nichtgermanische Personen. In jedem Fall wirft der Runenknochen von Břeclav neues Licht auf die frühmittelalterlichen Kulturkontakte zwischen Germanen und Slawen in Südmähren und Niederösterreich.

3. Dazu vor allem Düwel 1992: 97-98.

4. Dazu etwa Tejral 1976: 106; Wolfram 1995: 69; Bierbrauer 2001: 80. Zum Verbleib langobardischer Bevölkerungsgruppen im norddanubischen Raum bis zur Mitte des 6. Jahrhunderts ausführlich und grundlegend Tejral 2005:146-147, 174 und passim.

5. Die Zeichen auf dem Stein von Aš (Gierach 1925), und die Authentizität der Ritzungen auf der Miniaturaxt von Lipová u Šluknova (dt. Hainspach; ERF 19) ist fraglich.

6. Fibel von Aquincum (SG-2; RäF 7), Fibelpaar von Bezenye (SG-13; RäF 95) und Halbsäule von Breza (SG-19; RäF 5); dazu Nedoma 2006:109 f. Anm. 5. 


\section{Danksagung}

Diese Studie entstand als Teilprojekt des Forschungsvorhabens der Forschungsagentur Grantove agentura ČR The emergence of early medieval aristocracy in East-Central Europe: An archaeological-historical view (Project code 18-08646S).

\section{Literatur}

Bierbrauer, V. 2001. Langobarden: Archäologisches: Die Langob[arden] im 5. Jh. bis 568. Die Langob[arden] in Italien. Reallexikon der Germanischen Altertumskunde 18, 78-93.

Dostál, B. 1993. Kulturkontakte im mährisch-böhmisch-niederösterreichischen Raum während des Frühmittelalters (6.-10. Jahrhundert). In Th. Winkelbauer (Hrsg.), Kontakte und Konflikte. Böhmen, Mähren und Österreich. Aspekte eines Jahrtausends gemeinsamer Geschichte, 19-26. (Schriftenreihe des Waldviertler Heimatbundes 3.) Horn/Waidhofen an der Thaya: Waldviertler Heimatbund.

Düwel, K. 1992. Runen als magische Zeichen. P. Ganz (Hrsg.), Das Buch als magisches und als Repräsentationsobjekt, 87-100. Wiesbaden: Harrassowitz. - Wieder in R. Simek (Hrsg.), K. Düwel, Runica minora. Ausgewählte Schriften zur Runenkunde, 211-222. (Studia Medievalia Septentrionalia 23.) Wien: Fassbaender.

Düwel, K., Nedoma, R. \& Oehrl, S. (im Druck). Die südgermanischen Runeninschriften. 2 Bd.e. (RGA-Ergänzungsbd. 119.) Berlin \& Boston: de Gruyter.

ERF $[+$ Nr.] = H. Arntz \& H. Zeiss. 1939. Die einheimischen Runendenkmäler des Festlandes. (Gesamtausgabe der älteren Runendenkmäler 1.) Leipzig: Harrassowitz.

Gierach, E. 1925. Ein Stein mit Runen bei Asch? Sudeta 1. 145-146.

Jelínková, D. 1985. Doplňky k mapě nalezišt' s keramikou pražského typu na Moravě. Památky archeologické 76. 456-473.

Nedoma, R. 2006. Schrift und Sprache in den südgermanischen Runeninschriften. In A. Bammesberger \& G. Waxenberger (Hrsg.), Das fupark und seine einzelsprachlichen Weiterentwicklungen, 109-156. (RGA-Ergänzungsbd. 51.) Berlin \& New York: de Gruyter. https://doi.org/10.1515/9783110922981.109

Nowotny, E. 2015. Die früh- bis hochmittelalterliche Siedlung von Mitterretzbach, Niederösterreich. (Archäologische Forschungen in Niederösterreich N.F. 1.) Krems: Amt der Niederösterreichischen Landesregierung.

RäF [+ Nr.] = W. Krause \& H. Jankuhn. 1966. Die Runeninschriften im älteren Futhark. 2 Bd.e. (Abhandlungen der Akademie der Wissenschaften in Göttingen, Philolog.-Histor. Kl., 3. F., 65.) Göttingen: Vandenhoeck \& Ruprecht.

Szameit, E. 2000. Zum archäologischen Bild der frühen Slawen in Österreich. Mit Fragen zur ethnischen Bestimmung karolingerzeitlicher Gräberfelder im Ostalpenraum. In R. Bratož (Hrsg.), Slovenija in sosednje dežele med antiko in karolinško dobo. Začetki slovenske etnogeneze, 507-544. (Situla 39.) Ljubljana: Narodni muzej Slovenije.

Tejral, J. 1976. Grundzüge der Völkerwanderungszeit in Mähren. (Studie Archeologického Ústavu Akademie Věd ČR v Brně 4,2.) Praha: Academia. 
Tejral, J. 2005. Zur Unterscheidung des vorlangobardischen und elbgermanischlangobardischen Nachlasses. W. Pohl \& P. Erhart (Hrsg.), Die Langobarden. Herrschaft und Identität, 103-200. (Denkschriften der Österr. Akademie der Wissenschaften, Philosoph.-Histor. Kl., 329.)

Wawruschka, C. 2009. Frühmittelalterliche Siedlungsstrukturen in Niederösterreich.

(Mitteilungen der Prähistor. Kommission der Österr. Akademie der Wissenschaften 68.) Wien: Verlag der Österr. Akademie der Wissenschaften.

Wolfram, H. 1995 [= 2003]. Grenzen und Räume. Geschichte Österreichs vor seiner Entstehung. (Österreichische Geschichte 378-907 [4].) Wien: Ueberreuter.

\section{Korrespondenzanschriften}

Jiří Macháček

Ústav archeologie a muzeologie

Masarykova univerzita

Arna Nováka 1

CZ-6020o Brno

Czech Republic

machacek@phil.muni.cz

Robert Nedoma

Abteilung Skandinavistik

Institut für Europäische und Vergleichende

Sprach- und Literaturwissenschaft

Universität Wien

Universitätsring 1

A-1010 Wien

Austria

robert.nedoma@univie.ac.at 